ORIGINAL

\title{
Prevalência de lesões podais em bubalinos
}

\section{Prevalence of claw diseases in buffaloes}

\author{
Rinaldo Batista Viana ${ }^{1 *}$, Bruno Moura Monteiro², Waldjânio de Oliveira Melo², Daniel Rocha de Oliveira ${ }^{3}$, \\ Luciara Celi Chaves Daher ${ }^{1}$, José Dantas Ribeiro Filho ${ }^{4}$ \\ ${ }^{1}$ Instituto da Saúde e Produção Animal, Universidade Federal Rural da Amazônia (UFRA), Belém, PA, Brasil \\ 2 Universidade Federal Rural da Amazônia (UFRA), Paragominas, PA, Brasil \\ ${ }^{3}$ Agência Estadual de Defesa Agropecuária do Estado do Pará (ADEPARA), Santarém, PA, Brasil \\ ${ }^{4}$ Universidade Federal de Viçosa (UFV), Viçosa, MG, Brasil
}

\section{Resumo}

A criação de búfalos no mundo todo e, em particular no Brasil e países do Mercosul, vem se expandindo. Todavia, para obtenção de índices satisfatórios de produtividade, os animais necessitam de uma boa gestão da sanidade. Dentre as muitas doenças comuns aos bovinos, algumas se comportam diferentemente em bubalinos. Portanto, objetivou-se com esse estudo descrever a prevalência das enfermidades podais em bubalinos criados em áreas de várzea e campos alagados da Ilha de Marajó. 0 estudo foi realizado em matadouro localizado no município de Belém, utilizando-se 177 bubalinos (97 fêmeas e 80 machos) criados em lotação contínua em fazendas localizadas no arquipélago do Marajó. Os 708 cascos foram examinados individualmente, onde cada uma das 1416 unhas e 708 espaços interdigitais (2124 áreas dos cascos) foram classificados de acordo com a doenca apresentada. A análise estatística dos dados foi conduzida com base no teste de Qui-quadrado $\left(\mathrm{x}^{2}\right)$, com nível de significância de $5 \%$, a fim de verificar associação significativa entre sexo e cada uma das variáveis estudadas. 0 estudo mostrou que 14,12\% (25/177) dos búfalos avaliados apresentavam pelo menos uma lesão em uma das áreas dos cascos. Das 2124 áreas examinadas, 1,74\% (37/2124) apresentavam enfermidades podais. Tanto em machos como em fêmeas, a doença mais prevalente foi a unha em tesoura (UT), com $0,86 \%(10 / 1164)$ e 1,04\% (10/960) de unhas afetadas pela doença, respectivamente $(p=0,1571)$, sendo que 0,38\% (4/1062) das unhas com UT foram diagnosticadas nos membros anteriores, enquanto 1,51\% (16/1062) nos membros posteriores ( $\mathrm{p}=0,0414$ ). Considerando-se o plano mediano do corpo dos animais, 0,56\% (6/1062) das UT foram observadas nas unhas direitas e 1,32\% $(14 / 1062)$ nas esquerdas ( $p=0,0387)$. Os dados obtidos permitem observar que em bubalinos, tanto para machos quanto para fêmeas, a lesão mais prevalente é a UT, o que possivelmente está relacionado à ausência da prática de casqueamento corretivo.

Palavras-chave: Pododermatites. Casco. Unha. Doença. Bubalus bubalis bubalis. 


\section{Abstract}

The breeding of buffaloes worldwide, and in particular in Brazil and Mercosur countries, has been expanding. However, in order to obtain satisfactory levels of productivity, animals need good health management. Among the many diseases common to cattle, some behave differently in buffaloes. Therefore, this study aimed to describe the occurrence of hooves diseases in buffaloes raised in floodplain areas and flooded fields on the island of Marajó. This study was carried out in a slaughterhouse located in the city of Belém, Brazil, where 177 buffaloes (97 females and 80 males) from municipalities of the Marajó Island were used. Statistical analysis of the data was performed based on the chi-square test $\left(x^{2}\right)$, with a significance level of $5 \%$, in order to verify a significant association between sex and each of the variables studied. The study showed that $14.12 \%$ (25/177) of the evaluated buffalo had at least one lesion in one of the hull regions. of the 2,124 digits examined, 1.74\% (37/2124) presented with hooves diseases. In both males and females the most prevalent disease was the scissor claws (UT) with $0.86 \%$ (10/1.164) and 1.04\% (10/960) of claws affected by the disease, respectively ( $p=0.157)$. Since $0.38 \%(4 / 1,062)$ of the claws with UT were diagnosed in the anterior limbs while $1.51 \%(16 / 1,062)$ were in the hind legs $(p=0.0414)$. Considering the medial plane of the body of the animals, $0.56 \%(6 / 1,062)$ of the UT were obsed on the right claws and $1.32 \%(14 / 1,062)$ on the left $(p=0.0387)$. The data obtained allow us to observe that in buffalos the most prevalent lesion is a UT both for males and females, and that possibly this is related to the absence of the practice of hoof trimming.

Keywords: Pododermatitis. Hoof. Claw. Disease. Bubalus bubalis bubalis.

\section{Introdução}

O búfalo é bem adaptado a regiões alagadiças e terrenos inundáveis, como as condições das várzeas e campos alagados da Ilha de Marajó. A largura de seus cascos fendidos e as articulações apropriadas da espécie permitem menor esforço de sucção quando o animal se desloca em solos moles e lamacentos. Assim como os bovinos, os bubalinos dependem dos dígitos para a sua manutenção, em posição quadrupedal, para se alimentarem e reproduzirem, permitindo, assim, bons resultados na produção. Portanto, é fundamental que as extremidades dos membros locomotores estejam sempre saudáveis, permitindo o deslocamento dos animais, sobretudo nos sistemas extensivos e ultraextensivos (Greenough e Weaver, 1997; Nicoletti, 2003), característicos do arquipélago do Marajó.

Deve-se pesar ainda, os prejuízos causados por enfermidades podais, bem como a considerável diminuição na produção de leite e carne, descarte precoce de animais, baixa fertilidade causada pelo aumento no intervalo entre partos, produção de sêmen de baixa qualidade, altos custos em serviços veterinários e tratamentos (Nicoletti, 2003).

Embora as enfermidades digitais ocorram mundialmente, é preciso assinalar a lacuna de informações, dado o reduzido número de trabalhos científicos acerca das estruturas morfológicas dos cascos de diferentes espécies e raças criadas nas mesmas condições, bem como das enfermidades que os atingem (Mendonça et al., 2003). Tratando-se de ruminantes criados no trópico úmido amazônico, os dados são ainda mais escassos. Embora existam alguns estudos sobre as principais doenças que acometem os cascos e suas prevalências, sobretudo nas regiões alagadiças tropicais amazônicas (Oliveira etal., 2007; Silveira et al., 2008, 2009, 2010, 2011, 2018; Silva et al., 2015), a quase totalidade desses trabalhos obtidos na literatura coligida foi realizada em bovinos.

Objetivou-se, portanto, determinar a prevalência das enfermidades podais em bubalinos criados no arquipélago do Marajó, verificando qual das enfermidades podais apresenta maior prevalência, quais estruturas do casco são mais acometidas por afecções e qual a influência do sexo na prevalência das lesões podais.

\section{Materiais e métodos}

0 estudo foi realizado em matadouro localizado no município de Belém, utilizando-se 177 bubalinos (97 fêmeas e 80 machos). Os animais eram mantidos em lotação contínua em fazendas de criação 
tradicional localizadas no arquipélago do Marajó, na Ilha de Marajó, no estado do Pará (Tabela 1), destinados à produção de leite e carne.

Tabela 1 - Número de bubalinos estudados de acordo com 0 município de origem do animal

\begin{tabular}{lc}
\hline Município & Número de animais \\
\hline Santa Cruz do Arari & 22 \\
Muaná & 25 \\
Cachoeira do Arari & 27 \\
Chaves & 53 \\
Soure & 50 \\
\hline Total & 177 \\
\hline
\end{tabular}

Cada uma das 1416 unhas e 708 espaços interdigitais, perfazendo 2124 áreas dos cascos, foram analisados após o abate, examinando-se individualmente todas as áreas dos cascos (unhas e espaço interdigital) dos animais inlcuídos no estudo. Para classificação das doenças e lesões podais, a metodologia utilizada foi a proposta por Borges et al. (2017): unha assimétrica (UA); parede dorsal côncava (PC); unha em saca-rolha (SR); dermatite digital (DD) ou dermatite papilomatosa; dermatite interdigital (DI) ou superficial (frieira); sola dupla (SD); erosão de talão (ET) (podridão do casco); fissura da parede axial (FPA); fissura horizontal da parede (FHP); fissura vertical da parede (FVP); hiperplasia interdigital (HI); flegmão interdigital (FI) (panarício); unha em tesoura (UT) ; hemorragia difusa da sola (HDS); hemorragia circunscrita da sola (HCS); edema de coroa e/ou bulbo (ECB); úlcera de sola (US); úlcera de bulbo (UB); úlcera de pinça (UP); necrose de pinça (NP); sola fina (SF); fissura de linha branca (FlB); abscesso de linha branca (AlB); pododermatite séptica (broca, podridão do casco); pododermatite do paradígito (necrose da sobreunha, arranquio da sobreunha); tungíase (bicho-de-pé); e miíase (bicheira).

\section{Análise estatística}

Os dados de ocorrência de lesões foram apresentados como frequência $(\%, n / n)$. As ocorrências de lesões foram comparadas de acordo com as variáveis classificatórias: sexo, área do casco (unhas e espaço interdigital), classificação da doença, plano transversal (membros anteriores e posteriores), plano mediano (membros direito e esquerdo), unhas (lateral e medial), assim como as interações entre as mesmas.

A análise estatística foi realizada pelo teste de Quiquadrado $\left(\mathrm{x}^{2}\right)$, adotando-se nível de significância de $5 \%$, a fim de verificar associação significativa entre cada uma das variáveis estudadas.

\section{Resultados e discussão}

Apesar de Silva et al. (2015) reportarem que a espécie bubalina raramente apresenta enfermidades digitais, observou-se, após os exames de todos os cascos, que 14,12\% (25/177) dos búfalos avaliados apresentavam pelo menos uma lesão em uma das áreas do casco (unhas e espaços interdigitais), estando abaixo dos resultados encontrados $(17,7 \%)$ por Guccione et al. (2016) em búfalos Mediterrâneos criados em fazendas de exploração leiteira na Itália. Sabe-se que o desgaste do casco bubalino está diretamente correlacionado com condições climáticas (índice pluviométrico), independentemente da raça, espécie ou sistema de criação adotado. Por isso, animais criados em condições distintas podem apresentar diferentes prevalências de doenças podais (Túlio, 2006).

Estudando a morfometria do casco de bovinos das raças Nelore, Curraleira e Pantaneira e de bubalinos e sua relação com a etiopatogenia das enfermidades digitais, Silva e et al. (2015) inferem que embora as observações diárias indiquem que bubalinos são animais considerados mais resistentes às enfermidades podais, é necessário desenvolver estudo para comprovar tal fato. Necessário, também, comprovar se as características morfométricas do casco entre as distintas espécices, bovinos $\mathrm{x}$ bubalinos, poderão ser um fator de risco para as doenças digitais e contribuir para a gênese multifatorial de tais enfermidades. Tal assertiva de Silva et al. (2015) corrrobora as observações de Dirksen et al. (2005), Greenough (2007) e Lima (2011), ao considerarem as medidas morfométricas um fator de risco para as enfermidades podais nos bovinos. Silva et al. (2015) afirmam, ainda, que as 
diferenças observadas na morfometria dos dígitos de bubalinos e bovinos podem ajudar a explicar a menor ocorrência de doenças digitais nos bubalinos.

Dos 2124 dígitos examinados, 1,74\% (37/2124) apresentava enfermidades podais. Considerando-se o plano transversal do corpo dos animais, observouse que os membros mais acometidos por doenças podais foram os membros posteriores (Tabela 2), com maior ocorrência de lesões nestes membros do que nos anteriores $(\mathrm{p}=0,0311)$. Evidenciou-se, portanto, que $32,43 \%$ das lesões diagnosticadas acometeram os membros anteriores, enquanto $67,57 \%$ foram observadas nos membros posteriores (Figura 1). Já entre membros direitos (51,35\%) e esquerdos (48,65\%), não houve diferenca entre a ocorrência de lesões ( $p=0,8683$ ) (Figura 2).

Tabela 2 - Porcentagem e número de lesões podais em bubalinos criados em pastejo continuado no arquipelágo do Marajó, PA, de acordo com o membro acometido e sexo dos animais

\begin{tabular}{|c|c|c|c|c|c|c|}
\hline \multirow{3}{*}{ Variável } & \multicolumn{6}{|c|}{ Porcentagem e número de lesões podais } \\
\hline & \multicolumn{2}{|c|}{ Sem lesões } & \multicolumn{2}{|c|}{ Com lesões } & \multicolumn{2}{|c|}{ Total } \\
\hline & $\%$ & $\mathrm{n}$ & $\%$ & $\mathrm{n}$ & $\%$ & $\mathrm{n}$ \\
\hline Fêmeas & $53,91(98,37)$ & 1145 & $0,89(1,63)$ & 19 & $54,80(100)$ & 1164 \\
\hline Machos & $44,35(98,13)$ & 942 & $0,85(1,87)$ & 18 & $45,20(100)$ & 960 \\
\hline Total & 98,26 & 2087 & 1,74 & 37 & 100 & 2124 \\
\hline Anteriores & $49,44(98,87)$ & 1050 & $0,56(1,13)$ & 12 & $50,0(100)$ & 1062 \\
\hline Posteriores & $48,82(97,65)$ & 1037 & $1,18(2,35)$ & 25 & $50,0(100)$ & 1062 \\
\hline Total & 98,26 & 2087 & 1,74 & 37 & 100 & 2124 \\
\hline Direitos & $49,11(98,21)$ & 1043 & $0,89(1,79)$ & 19 & $50,0(100)$ & 1062 \\
\hline Esquerdos & $49,15(98,31)$ & 1044 & $0,85(1,69)$ & 18 & $50,0(100)$ & 1062 \\
\hline Total & 98,26 & 2087 & 1,74 & 37 & 100 & 2124 \\
\hline
\end{tabular}

Nota: Qui-quadrado: Machos $x$ fêmeas, $p=0,6705$. Membros anteriores $x$ membros posteriores, $p=0,0311$. Membros direitos $x$ membros esquerdos, $p=0,8683$. Valores entre parênteses consideram o percentual em função das lesões em cada membro.

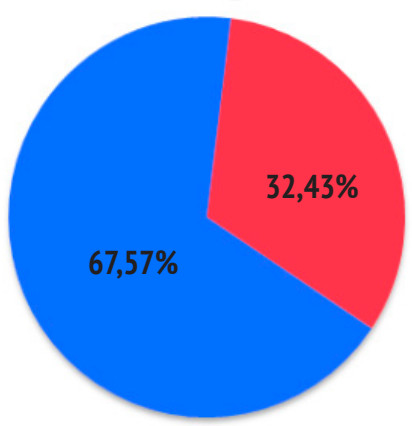

Membros anteriores

Membros posteriores

Figura 1 - Porcentagem de lesões podais nas unhas e espaços interdigitais em bubalinos criados em pastejo continuado no arquipélago do Marajó, PA, de acordo com plano transversal do corpo dos animais (membros anteriores $\mathrm{x}$ membros posteriores).

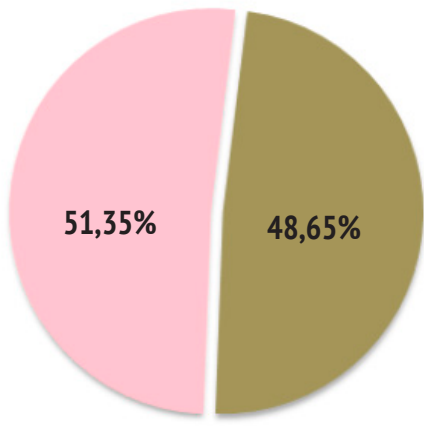

Membros esquerdos Membros direitos

Figura 2 - Porcentagem de lesões podais nas unhas e espaços interdigitais em bubalinos criados em pastejo continuado no arquipélago do Marajó, PA, de acordo com o plano mediano do corpo dos animais (membros esquerdos $\mathrm{x}$ membros direitos). 
Guccione et al. (2016) também verificaram maior frequência de distúrbios podais nos membros pélvicos $(73,8 \%, 169 / 229)$ em relação aos mebros torácicos $(26,2 \%, 60 / 229)$ de bubalinos Mediterrâneos leiteiros, sendo que 7,8\% (16/205) localizava-se tanto nos membros dianteiros quanto nos posteriores. De acordo com Borges (1998), o maior acometimento dos membros posteriores pode ocorrer pelo maior contato com fezes, urina e excesso de umidade.

As unhas são mais acometidas do que o espaços interdigitais, sendo que a ocorrência de lesões nas unhas foi de 2,19\% (31/1416) e nos espaços interdigitais foi de $0,85 \%$ (6/708), culminando

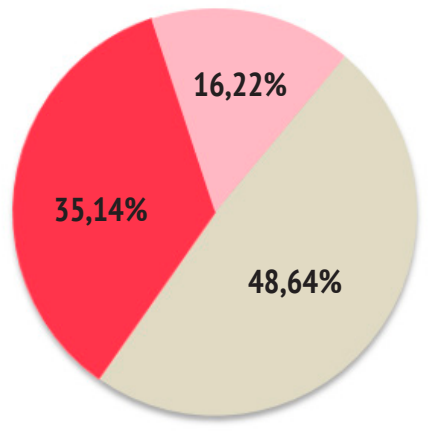

Unhas laterais Unhas mediais Espaços interdigitais

Figura 3 - Porcentagem de lesões podais em bubalinos criados em pastejo continuado no arquipélago do Marajó, $\mathrm{PA}$, de acordo com a área do casco afetada.

Das unhas examinadas nas búfalas (Tabela 2), 1,63\% (19/1164) apresentava lesões, enquanto nos machos esse valor foi igual a 1,87\% (18/960). Não se evidenciou, portanto, variação na ocorrência de lesões em função do sexo $(p=0,6705)$. Ao se considerar o porcentual de unhas examinadas, de todas as unhas com lesões podais, 0,89\% (19/2124) foram obervadas nas búfalas, enquanto $0,85 \%$ (18/2124) foram diagnosticadas nos machos. Portanto, 54,80\% do total de unhas acometidas por algum tipo de doença podal foram diagnosticadas nas búfalas e $45,20 \%$ nos machos.

Tanto em machos como em fêmeas, a doença mais prevalente foi a UT, com $0,86 \%(10 / 1164)$ e $1,04 \%$ (10/960) de unhas afetadas pela doença, em $83,78 \%$ das lesões nas unhas e $16,22 \%$ nos espaços interdigitais ( $p=0,0259)$. Entre as unhas, tem-se as laterais com maior incidência de lesões $(2,54 ; 18 / 708)$, frente às unhas mediais $(1,85 \%$; $13 / 708)$, em que $48,65 \%$ das lesões ocorreram nas unhas laterais e $35,14 \%$ nas mediais ( $\mathrm{p}=$ 0,0499 ) (Figura 3). Nas unhas posteriores ocorreu o dobro de lesões em relação às anteriores (Figura 4), todavia não houve diferença entre as unhas laterias e mediais em cada casco, tanto anterior quanto posterior ( $\mathrm{p}=0,2616)$ (Tabela 3). Esse comportamento é similar ao de bovinos, em que as unhas mais acometidas são as laterais dos membros posteriores (Silveira et al., 2018).

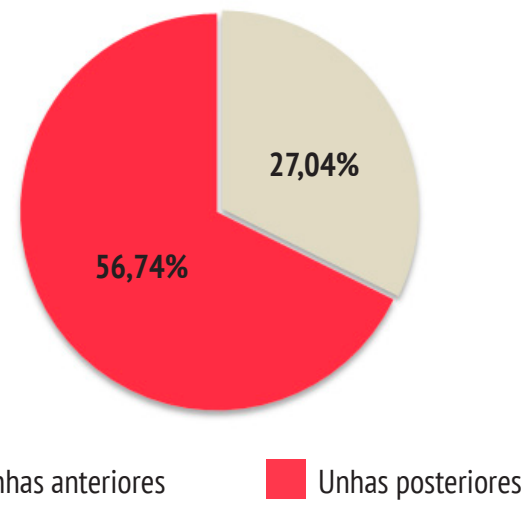

Figura 4 - Porcentagem de lesões podais em bubalinos criados em pastejo continuado no arquipélago do Marajó, PA, de acordo com a unha afetada.

respectivamente ( $\mathrm{p}=0,1571)$, sendo $0,38 \%$ (4/ 1062) diagnosticadas nos membros anteriores e $1,51 \%(16 / 1062)$ nos membros posteriores $(p=0,0414)$. Considerando-se o plano medial do corpo dos animais, 0,56\% (6/1062) das UT foram obsrevadas nas unhas direitas e 1,32\% (14/1062) nas unhas esquerdas ( $p=0,0387)$. Guccione et al. (2016) verificaram que as doenças relacinadas à disfunção do crescimento também foram as mais prevalentes (UA esteve presente em 17,0\% e SR em 15,8\% dos bubalinos observados; 220 e 205/1297, rspectivamente) e descreveram, ainda, casos de FI em $0,9 \%$ (12/1297), ALB em 0,8\% (11/1297), DD em $0,1 \%(1 / 1297)$ e IH em $0,1 \%(1 / 1297)$ dos animais investigados. 
As diferentes prevalências obtidas no presente estudo e no trabalho descrito por Guccione et al. (2016) podem estar relacionadas ao sistema de criação, visto que os búfalos criados na Ilha de Marajó vivem em áreas de várzea, enquanto na Itália os animais são criados em condições intensivas.
Outros fatores como a baixa produção leiteira das búfalas no Brasil, alimentação dos animais a pasto sem suplementação proteico-energética na maioria das fazendas e diferenças morfométricas podem contribuir para a ainda baixa prevalência das doenças podais em bubalinos.

Tabela 3 - Porcentagem de lesões podais em bubalinos criados em pastejo continuado no arquipelágo do Marajó, PA, de acordo com a região do casco afetada

\begin{tabular}{lcccccc}
\hline & \multicolumn{6}{c}{ Porcentagem e número de lesões podais } \\
\cline { 2 - 7 } Área do casco & \multicolumn{2}{c}{ Sem lesões } & \multicolumn{2}{c}{ Com lesões } & \multicolumn{3}{c}{ Total } \\
\cline { 2 - 6 } & $\%$ & $n$ & $\%$ & $n$ & $\%$ & $n$ \\
\cline { 2 - 7 } UADL & $8,15(97,74)$ & 173 & $0,19(2,26)$ & 4 & $8,33(100)$ & 177 \\
UADM & $8,24(98,87)$ & 175 & $0,09(1,13)$ & 2 & $8,33(100)$ & 177 \\
UAEL & $8,24(98,87)$ & 175 & $0,09(1,13)$ & 2 & $8,33(100)$ & 177 \\
UAEM & $8,24(98,87)$ & 175 & $0,09(1,13)$ & 2 & $8,33(100)$ & 177 \\
UPDL & $8,05(96,61)$ & 171 & $0,28(3,39)$ & 6 & $8,33(100)$ & 177 \\
UPDM & $8,15(97,74)$ & 173 & $0,19(2,26)$ & 4 & $8,33(100)$ & 177 \\
UPEL & $8,05(96,61)$ & 171 & $0,28(3,39)$ & 6 & $8,33(100)$ & 177 \\
UPEM & $8,10(97,18)$ & 172 & $0,24(2,82)$ & 5 & $8,33(100)$ & 177 \\
EIAD & $8,24(98,87)$ & 175 & $0,09(1,13)$ & 2 & $8,33(100)$ & 177 \\
EIAE & $8,33(100,0)$ & 177 & $0,00(0,00)$ & 0 & $8,33(100)$ & 177 \\
EIPD & $8,29(99,44)$ & 176 & $0,04(0,56)$ & 1 & $8,33(100)$ & 177 \\
EIPE & $8,19(98,31)$ & 174 & $0,14(1,69)$ & 3 & $8,33(100)$ & 177 \\
\hline Total & 98,26 & 2087 & 17474 & 37 & 100 & 2124 \\
\hline
\end{tabular}

Nota: Qui-quadrado: $p=0,2616$. Valores entre parênteses consideram o percentual em função das lesões em cada membro. UADL - unha anterior direita lateral; UADM - unha anterior direita medial; UAEL - unha anterior esquerda lateral; UAEM - unha anterior esquerda medial; UPDL - unha posterior direita lateral; UPDM - unha posterior direita medial; UPEL - unha posterior esquerda lateral; UPEM - unha posterior esquerda medial; EIAD - espaço interdigital anterior direito; EIAE - espaço interdigital anterior esquerdo; EIPD - espaço interdigital posterior direito; EIAE - espaço interdigital posterior esquerdo.

\section{Conclusão}

Os dados obtidos permitem observar que em bubalinos a lesão mais prevalente é a unha em tesoura, tanto para machos quanto para fêmeas, o que possivelmente está relacionado a alguns fatores como ausência da prática de casqueamento corretivo, tipo do piso e sistema de criação. A prevalência de lesões podais obtidas é um alerta para a adoção de medidas de biosseguridade, visando a saúde dos cascos dos búfalos, pois com o aumento dos desafios sanitários e intensificação dos processos de criação da espécie, pode-se passar a ter uma maior ocorrência de doenças podais nas búfalas, como já ocorre em países onde a atividade é mais tecnificada.

\section{Referências}

Borges JRJ, Câmara ACL, Moscardini ARC, Rodrigues CA, Pitombo CA, Graça FAS, et al. Doenças dos dígitos dos bovinos: nomenclatura padronizada para o Brasil. Revista CFMV. 2017;23(73):45-52. 
Borges NC. Caracterização do fluido rumenal e dos parâmetros clínicos-laboratoriais de bovinos com pododermatite [dissertação]. Goiânia: Universidade Federal de Goiás; 1998. 69 p.

Dirksen G, Gründer HD, Stöber M. Medicina interna y cirugía del bovino. 4 ed. Buenos Aires: Inter-Médica Editorial; 2005. 1172 p.

Greenough PR. Bovine Laminitis and Lameness - A handson Approach. Philadelphia: Saunders; 2007. 311 p.

Greenough PR, Weaver AD. Lameness in cattle. Philadelphia: W.B. Saunders; 1997. 336 p.

Guccione J, Carcasole C, Alsaaod M, D’Andrea L, Di Loria A, De Rosa A, et al. Assessment of foot health and animal welfare: clinical findings in 229 dairy Mediterranean Buffaloes (Bubalus bubalis) affected by foot disorders. BMC Vet Res. 2016;12(1):107.

Lima FB. Morfologia e Morfometria dos cascos de bovinos Nelorados [dissertação]. Brasília: Universidade de Brasília; 2011. 86 p.

Mendonça AC, Silva LAF, Fioravanti MCS, Moraes JOR, Almeida CF, Oliveira KS, et al. Aspectos morfológicos dos dígitos de bovinos das raças Gir e Holandesa. Ci Anim Bras. 2003;4(1):53-60.

Nicoletti JLM. Manual de Podologia Bovina. São Paulo: Editora Manole; 2003.126 p.

Oliveira DR, Viana RB, Araújo CV, Monteiro BM. Ocorrência de lesões podais em bovinos de corte e bubalinos abatidos no Estado do Pará. Arch Vet Sci. 2007;12(4):38-40.

Silva LAF, Campos SBS, Rabelo RE, Vulcani VAS, Noronha Filho ADF, Freitas SLR. Análise comparativa da morfometria do casco de bovinos das raças Nelore, Curraleira e Pantaneira e de bubalinos e sua relação com a etiopatogenia das enfermidades digitais. Pesq Vet Bras. 2015;35(4):377-84.
Silveira JAS, Albernaz TT, Oliveira CMC, Duarte MD, Barbosa JD. Afecções podais em vacas da bacia leiteira de Rondon do Pará. Pesq Vet Bras. 2009;29(11):905-90.

Silveira JAS, Albernaz TT, Sousa MGS, Campos KF, Silva NS, Oliveira CMC, et al. Prevalência e características clínicas das enfermidades podais diagnosticadas pela central de diagnóstico veterinário (Cedivet), no estado do Pará, durante o período de 2000 a 2006. Encontro Nacional de Diagnóstico Veterinário; 28 jun - 03 jul 2008; Campo Grande, MS. 2008. Campo Grande: Endivet; 2008. p. 79-80.

Silveira JAS, Oliveira CMC, Albernaz TT, Silva NS, Vinhote MMS, Lima DHS, et al. Dermatite digital em bovinos de corte e leite criados em regime extensivo no estado do Pará. Encontro Nacional de Diagnóstico Veterinário; 25 28 out 2010; Campo Grande, MS. Intebrio. 2010;4(1);112.

Silveira JAS, Silva NS, Albernaz TT, Bomjardim HA, Andrade SJT, Oliveira CMC, et al. Afecções podais em bovinos associado à sodomia. Vet Zootec. 2011;18(4, Supl 3):294-7.

Silveira JAS, Silva NS, Albernaz TT, Bomjardim HA, Reis ASB, Oliveira CMC, et al. Epidemiological and clinical study of foot diseases in beef cattle extensive management in southeastern Pará, Brazil. Pesq Vet Bras. 2018;38(3):367-73.

Túlio LM. Estudo biométrico do casco bovino e bubalino: avaliação de características anátomo-fisiológicas do casco sadio [dissertação]. Curitiba: Universidade Federal do Paraná; 2006. 97 p. 